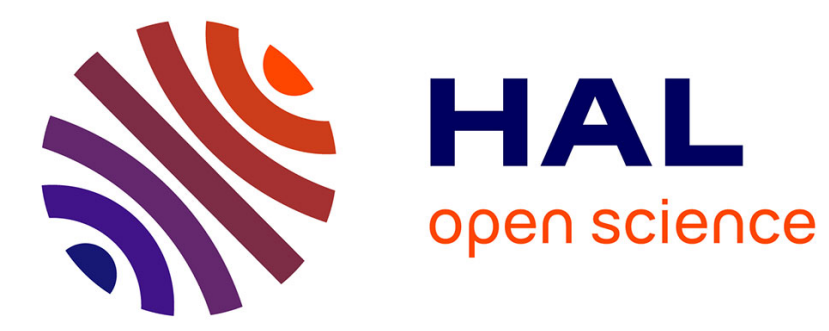

\title{
Node dynamics and cusps size distribution at the border of liquid sheets
}

E. Villermaux, Christophe Almarcha

\section{To cite this version:}

E. Villermaux, Christophe Almarcha. Node dynamics and cusps size distribution at the border of liquid sheets. Physical Review Fluids, 2016, 1 (4), pp.041902. 10.1103/PhysRevFluids.1.041902 . hal-03566843

\section{HAL Id: hal-03566843 \\ https://hal.science/hal-03566843}

Submitted on 11 Feb 2022

HAL is a multi-disciplinary open access archive for the deposit and dissemination of scientific research documents, whether they are published or not. The documents may come from teaching and research institutions in France or abroad, or from public or private research centers.
L'archive ouverte pluridisciplinaire HAL, est destinée au dépôt et à la diffusion de documents scientifiques de niveau recherche, publiés ou non, émanant des établissements d'enseignement et de recherche français ou étrangers, des laboratoires publics ou privés. 


\title{
Node dynamics and cusps size distribution at the border of liquid sheets
}

\author{
E. Villermaux ${ }^{1,2, *}$ and C. Almarcha ${ }^{1}$ \\ ${ }^{1}$ Aix Marseille Université, CNRS, Centrale Marseille, IRPHE UMR 7342, 13384 Marseille, France \\ ${ }^{2}$ Institut Universitaire de France, 75005 Paris, France
}

(Received 26 May 2016; published 29 August 2016)

\begin{abstract}
We study the intrinsic dynamics of cusps, or indentations, moving along a liquid sheet border, and characterize their ensemble statistics. Gordillo and collaborators [J. Fluid Mech. 754, R1 (2014)] have shown that the symmetrical stationary cusp is the only structure accommodating for both mass and momentum conservation at a steadily receding liquid sheet rim. Cusps are also known to typically move along a sheet border, to present an asymmetry, and to be distributed in size around a mean. We show here why a heterogeneous assembly of cusps traveling along the sheet rim occurs spontaneously, why big and small cusps coexist at the same time, and, more precisely, we establish a specific link between the microscopic dynamics directing their motion, and the ensemble averaged distribution of their sizes.
\end{abstract}

DOI: 10.1103/PhysRevFluids.1.041902

\section{INTRODUCTION}

All natural macroscopic objects are distributed in size. Because it typically results from an elaboration process ruled by a large number of independent factors, any cohesive object made from the aggregation of many elementary pieces will be more or less tall, wide, or thick around a mean; the fluctuations about the mean are described quantitatively by the central limit theorem [1], once the construction process of the object is known.

We are interested here in liquid sheets, and especially in their border, which has been known to be indented, as seen in Fig. 1, since the seminal contributions of Savart [2]. The border of a liquid sheet recedes because it is pulled by an unbalanced surface force at its rim and, even if initially smooth, develops intrinsic instabilities distorting it [3]. The instability eventually reaches a saturated state in the form of characteristic cusps [4-6], a phenomenon common with other related configurations involving free surface flows [7-10], or self-propagating fronts such as in flames $[11,12]$. The reason for the existence of these natural cusps, which do not require any external stimuli [13], nor any particular rheological feature of the liquid [14,15] to exist, was understood by Gordillo and collaborators [16]. Correcting a naive view of the rim recession dynamics proposed by Taylor [4] (see also Refs. [17-19] for successive flawed attempts, commented on in Ref. [20]), Gordillo et al. have shown that these cusps are the only structures accommodating for both mass and momentum conservation balances at a steady liquid sheet rim expelling ligaments and drops.

It is also known that these cusps are not stationary, moving along the sheet border, and that their size fluctuates around a mean, a feature which has yet to be explored. The subject of the present Rapid Communication is a discussion about why cusps spontaneously travel along the sheet rim, why big and small cusps coexist at the same time, and, more precisely, to establish a specific link between the microscopic dynamics directing their evolution, and the ensemble averaged distribution of their sizes.

\section{NODE EQUILIBRIUM AND DRIFT VELOCITY}

\section{A. Setup and basic relationships}

We want to study the intrinsic dynamics of cusps moving along a liquid sheet border, and characterize their size distribution. In order to address these questions, we make use of the configuration

*emmanuel.villermaux@univ-amu.fr 


\section{E. VILLERMAUX AND C. ALMARCHA}
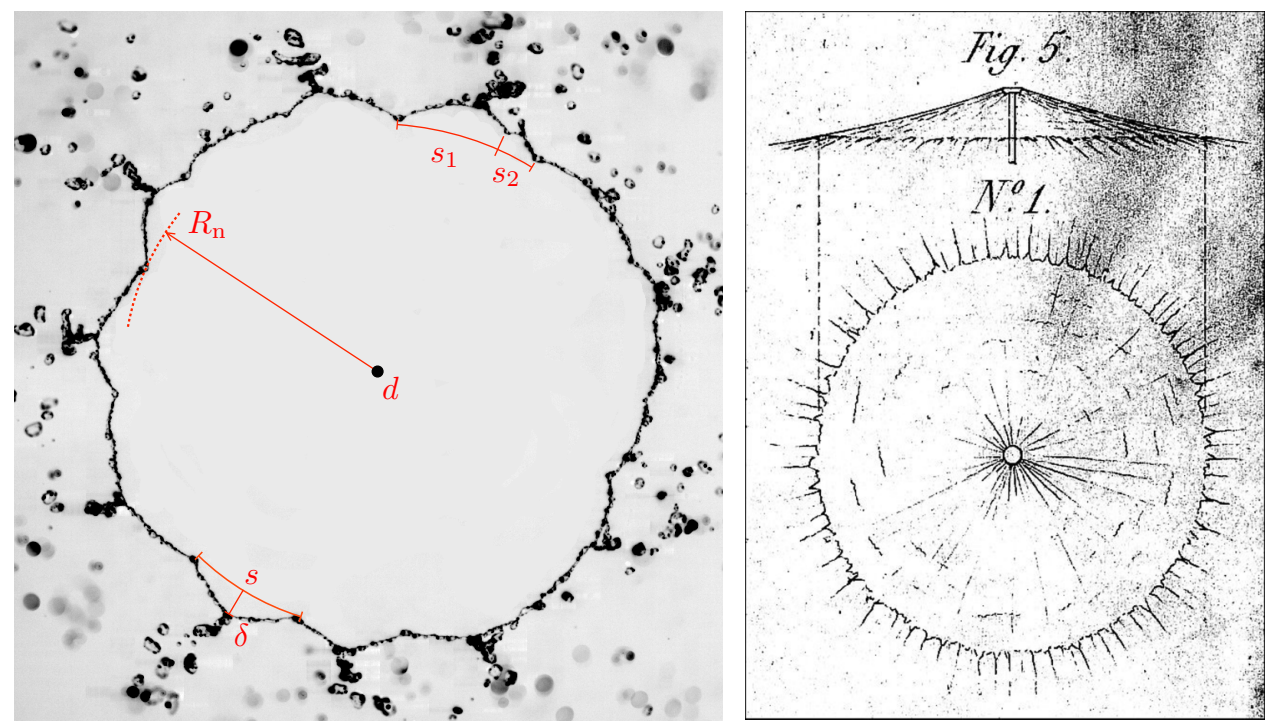

FIG. 1. Left: Snapshot of a water Savart sheet seen from above, exhibiting a typical cusp structure at the edge. The flow $\mathbf{u}$ is radial from the central jet (dark spot with diameter $d=3 \mathrm{~mm}$ ) with $\mathrm{We}=453$. The cusps are cells separated by nodes whose radial location is in $R_{\mathrm{n}}=(4 / 5) R_{\mathrm{TC}}$, where $R_{\mathrm{TC}} / d=\mathrm{We} / 16$ is the Taylor-Culick radius. Adjacent nodes define cells, with variable sizes $s$ around a mean $\langle s\rangle$. Nodes may move in an orthoradial direction, possibly merging with their neighbors, with this event defining the coalescence of adjacent cells: Here two cells with sizes $s_{1}$ and $s_{2}$ are in the process of merging. The height of a cusp is $\delta$. Right: The drawing made by Savart [2], by naked eye observation. Note the presence of sheet rim indentations, which are, however, too many, and the shape of the cusps, which is inverted from their real shape.

of a liquid jet impacting normally onto a solid disk, an extremely convenient "laboratory" proposed by Savart [2] to understand the consequences of the cohesion of liquids on the macroscopic world. At impact, the jet deviates in an axisymmetrical fashion and feeds a radially expanding sheet which is bordered, at some distance from the impact location, by a rim collecting the liquid. The rim is composed of a series of adjacent cusps separated by nodes (Fig. 1). The injected liquid is conveyed along the cusps towards their tip, where it is expelled in the form of corrugated ligaments, further destabilizing and producing droplets.

In absence of an interaction with ambient air (as long as We $\lesssim 10^{3}$; see Ref. [6]), and for a nonviscous liquid such as water, the only control parameters defining the expanding sheet geometry are the impacting jet diameter $d$ and the Weber number

$$
\mathrm{We}=\frac{\rho u^{2} d}{\gamma}
$$

where $u=|\mathbf{u}|$ is the norm of the radial velocity $\mathbf{u}, \rho$ the density of the liquid, and $\gamma$ its surface tension. The radial location of the nodes $R_{\mathrm{n}}$ is [16]

$$
R_{\mathrm{n}}=\frac{4}{5} R_{\mathrm{TC}}, \quad \text { with } \quad \frac{R_{\mathrm{TC}}}{d}=\frac{\mathrm{We}}{16},
$$

where $R_{\mathrm{TC}}$ denotes the Taylor-Culick [4,21] radius.

At the node, the border has a characteristic $\mathrm{V}$ shape, which is understood as follows: The node absorbs an incoming mass flow rate $q$ and momentum $q u$ which are split in the two deflected jets defining the cusp border. The deflection is due to the action of a capillary force $f$ acting perpendicular to the border, and to the recoil force of the exiting momenta of the deflected jets. Energy is not conserved. For a symmetrical cusp with angles $\theta$ as shown in Fig. 2(b), the velocity in 
(a)

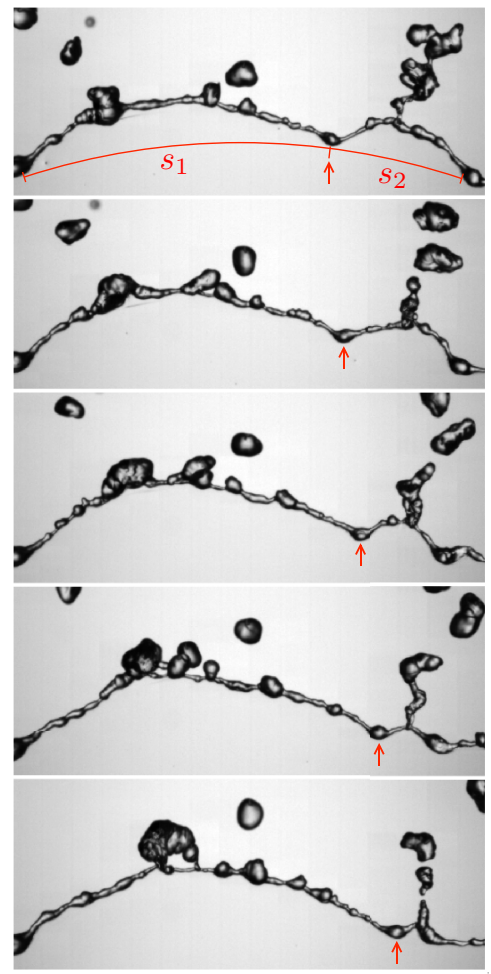

(b)

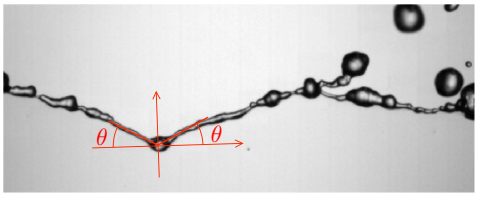

(c)

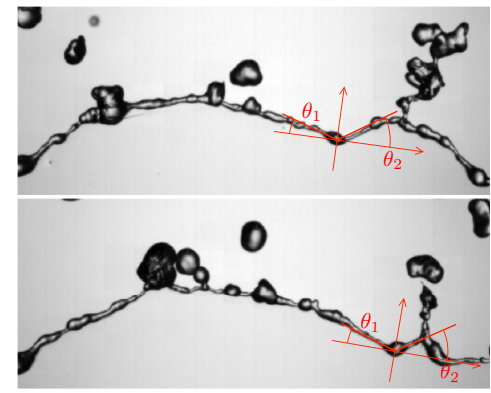

(d)

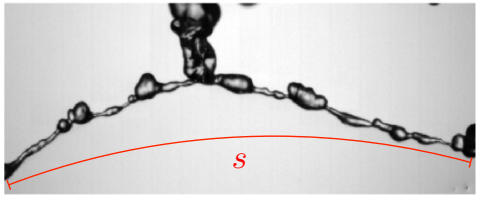

FIG. 2. An example of cusp dynamics and cell fusion obtained at $|\mathbf{u}|=3.07 \mathrm{~m} / \mathrm{s}$ and $\mathrm{We}=405$. The height of each image is $12.5 \mathrm{~mm}$, and are displayed every $3.5 \mathrm{~ms}$. (a) Fusion of two cells of sizes $s_{1}$ and $s_{2}$ through the motion of the dissymmetrical node (marked by an arrow) separating them, moving along the circle of radius $R_{\mathrm{n}}$ (see Fig. 1). (b) A steady symmetrical node with ejection angles both equal to $\theta=\arctan (1 / 2) \approx 26.6^{\circ}$. The upward arrow points in the direction of $\mathbf{u}$. (c) The ejection angles $\theta_{1}<\theta_{2}$ in sequence (a) are such that the node is propelled from left to right $\left(\theta_{1} \approx 20^{\circ}, \theta_{2} \approx 32^{\circ}\right.$ on the displayed portion of the node trajectory). (d) The final cell of sequence (a) after coalescence, with size $s=s_{1}+s_{2}$.

the jets is $u \sin \theta$, and the equilibrium perpendicular to the sheet at the node location reads

$$
q u=2 \times \frac{q}{2} u \sin ^{2} \theta+f
$$

or

$$
\frac{f}{q u}=1-\sin ^{2} \theta .
$$

The condition of a stationary node also imposes [16]

$$
\tan \theta=\frac{1}{2}, \text { that is, } \theta \approx 26.6^{\circ},
$$

a value which was confirmed to hold in the mean for a large collection of nodes by Gordillo et al., within noticeable fluctuations, however. These variations are natural, as we will see, and are contingent to the observed fact that nodes are typically nonsymmetrical, with two different ejection angles, and are moving along the sheet border.

\section{B. Drift velocity}

For a nonsymmetrical cusp, equilibrium is achieved when the node travels at a nonzero constant velocity $\mathbf{v}$ with norm $v=|\mathbf{v}|$ along the sheet border, in a direction perpendicular to the incoming 


\section{E. VILLERMAUX AND C. ALMARCHA}

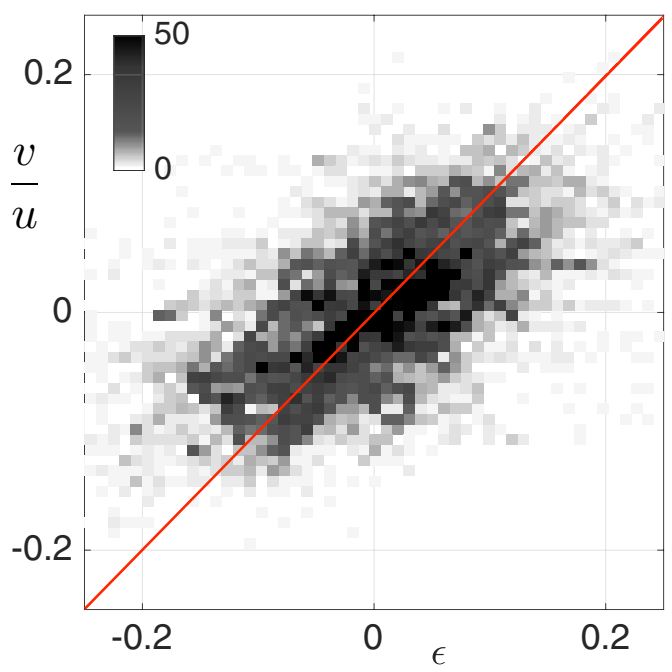

FIG. 3. Stacked histogram of the joined measurement of the drift velocity $v$ and dissymmetry $\epsilon$ (in radiants) of 13170 nodes at We $=453$ in the conditions of Fig. 1. The darkness of each discretizing pixel of the $\{v, \epsilon\}$ plane is proportional to the number of recorded events (white for zero, and black for 50 or more). Most of the occurrences concentrate around $\epsilon=0$ and stretch, with some scatter, along the line (in red) expected from the deterministic Eq. (9).

flow, so that, at the node, $\mathbf{u} \cdot \mathbf{v}=0$. If $\theta_{1}$ and $\theta_{2}$ are now the deflection angles of the side jets [Fig. 2(c)], and assuming that the flow rates remain equally split as in the symmetrical equilibrium (the pressure inside the node bulge is not altered by a tilt of the ejection angles), the stationary force balance in Eq. (3) becomes

$$
q u=\frac{q}{2}\left(v \cos \theta_{1}+u \sin \theta_{1}\right) \sin \theta_{1}+\frac{q}{2}\left(-v \cos \theta_{2}+u \sin \theta_{2}\right) \sin \theta_{2}+f,
$$

which, making use of (4), provides

$$
\frac{v}{u}=\frac{2 \sin ^{2} \theta-\left(\sin ^{2} \theta_{1}+\sin ^{2} \theta_{2}\right)}{\cos \theta_{1} \sin \theta_{1}-\cos \theta_{2} \sin \theta_{2}} .
$$

We seek for a slight tilt of the ejection directions by an angle $\epsilon$ with respect to the symmetrical one $\theta$ on both sides of the node,

$$
\theta_{1}=\theta-\epsilon \text { and } \theta_{2}=\theta+\epsilon .
$$

This choice is arbitrary, since the departures from $\theta$ on each side of the node need not be linked to each other. This is a convenient though realistic choice [see Fig. 2(c)], allowing for a transparent discussion. Indeed, from (7), we anticipate that

$$
\frac{v}{u}=\tan \epsilon
$$

independent of $\theta$. The drift velocity $v$ is zero when $\epsilon=0$ for a symmetrical cusp, and has the sign of $\epsilon$; it is positive when $\theta_{2}$ is larger than $\theta_{1}$, as expected. The analysis of a large collection of nodes in the conditions of Fig. 1 reported in Fig. 3 shows that the above relation holds, in the mean, to indicate a clear correlation between the tilt angle $\epsilon$ and the drift $v$ among an otherwise noisy dependence. The noise comes mainly from the measurement uncertainty of the angles at the nodes in the broad field images such as in Fig. 1, but it has also a physical origin: It reflects the constant nucleation of new cusps on already tilted portions of the rim, not described by Eq. (9). It is only when these newly formed cusps have a chance to relax towards the close-to-symmetrical shape on a nontilted portion 


\section{NODE DYNAMICS AND CUSPS SIZE DISTRIBUTION AT ...}

(a)

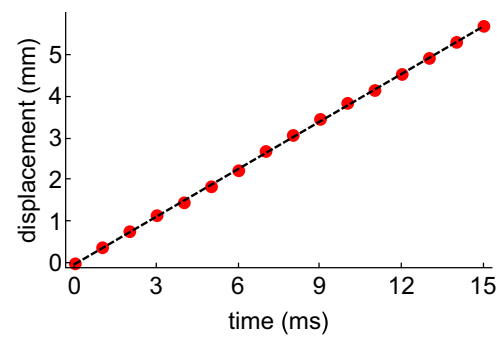

(b)

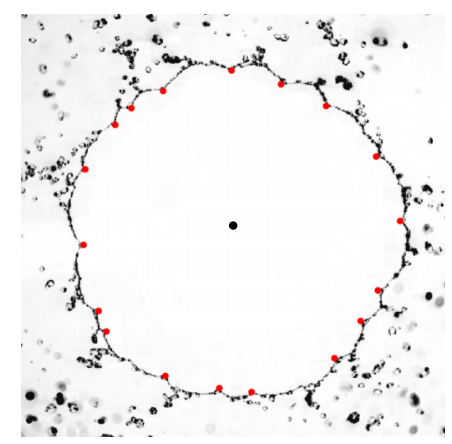

(c)

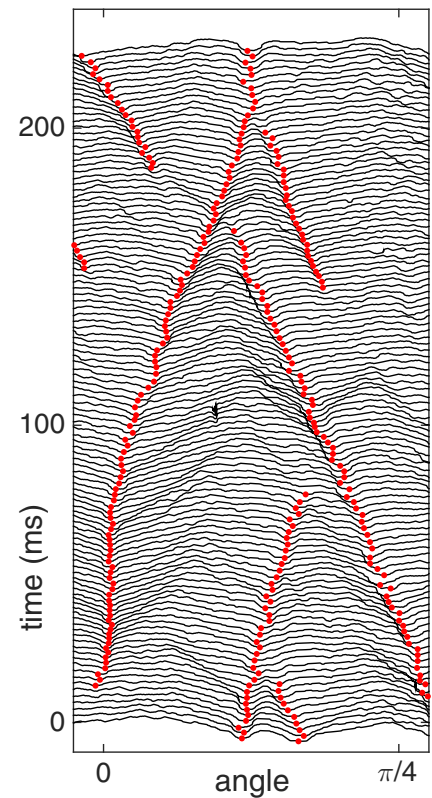

FIG. 4. (a) Trajectory of the node in Fig. 2(a) with ejection angles $\theta_{1} \approx 20^{\circ}$ and $\theta_{2} \approx 32^{\circ}$, compatible with $\epsilon=7^{\circ}$, anticipating, from Eq. (9), a drift velocity $v=0.37 \mathrm{~m} / \mathrm{s}$; the measured velocity is $0.38 \mathrm{~m} / \mathrm{s}$. (b) Detection of the nodes (red dots) around the sheet at a given instant of time. (c) Space-time diagram showing the evolution of the node location in a fixed angular sector covering $1 / 8$ of the sheet perimeter $2 \pi R_{\mathrm{n}}$ at the node radius $R_{\mathrm{n}}$. Successive fronts are separated by $2 \mathrm{~ms}$. Node drift, merging, and spontaneous nucleation are evident.

of the rim that Eq. (9) operates. Most of cusps do so, explaining why the region around $\epsilon=0$ is the densest on the event histogram in Fig. 3.

On focused fields, the measurement of the angles is less ambiguous: Figure 4(a) shows the trajectory of the node in Fig. 2(a) whose ejection angles are $\theta_{1} \approx 20^{\circ}$ and $\theta_{2} \approx 32^{\circ}$ [Fig. 2(c)], values which are compatible with $\epsilon \approx 7^{\circ}$. The corresponding drift velocity $v$ from Eq. (9) with $u=3.07 \mathrm{~m} / \mathrm{s}$ is $v=0.37 \mathrm{~m} / \mathrm{s}$ while the measured velocity is $0.38 \mathrm{~m} / \mathrm{s}$ on the corresponding portion of the trajectory.

\section{TIME SCALES AND CELL SIZE DISTRIBUTION}

Nodes separating two adjacent cells with uneven sizes disappear by merging with their nearest neighbor, hence erasing the smallest cell at the expense of the larger one. Two adjacent cells with sizes $s_{1}$ and $s_{2}$ will thus give birth to a larger cell whose size is such that [see, e.g., Fig. 2(d)]

$$
s=s_{1}+s_{2} .
$$

Nodes are, concomitantly, constantly renewed at the border of the sheet by the capillary destabilization of its rim, so that there are, in the mean, of the order of $N=10-20$ cells, or cusps, at the border of the sheet. We have checked that node nucleation occurs randomly along the sheet rim so that $s_{1}$ and $s_{2}$ are independent of each other when they merge, according to (10).

Let $P(s)=p(s /\langle s\rangle) /\langle s\rangle$ be the distribution of cell sizes along the sheet. Previous studies in different but related contexts, where an addition rule such as (10) applies, have shown that the shape of the distribution is of a Gamma type,

$$
p(x=s /\langle s\rangle)=\frac{v^{v}}{\Gamma(\nu)} x^{\nu-1} e^{-v x} .
$$




\section{E. VILLERMAUX AND C. ALMARCHA}

May it be for scalar mixtures stirred randomly [22], for the drops formed from ligament breakups [23], or for the fragmentation of simple solids [24,25], when an addition rule between uncorrelated variables (scalar concentrations, liquid ligament corrugations, clusters of atoms) holds, it implies that their distribution is of the type in Eq. (11). This distribution is stable by self-convolution, the operation which corresponds in probability space to the addition in variable space [1].

Furthermore, it was shown that the only parameter in Eq. (11), namely, the order $v$ setting the width of the distribution around its mean, is related to the microscopic dynamics of the variable addition process itself. This way, the incomplete convolution theory [24] relates the microscopic kinetics with the global statistics. In the present problem, the determination of $v$ amounts to a comparison of the coalescence time of two cells $t_{s_{1}, s_{2}}$ with the mean field coalescence time $t_{\langle s\rangle-\sigma,\langle s\rangle+\sigma}$ of two "typical" cells having a size given by the mean size, plus or minus a fluctuation of the order of the standard deviation $\sigma$ of the size distribution, namely,

$$
s_{1}=\langle s\rangle+\sigma \quad \text { and } \quad s_{2}=\langle s\rangle-\sigma .
$$

The quantity which is central to the discussion of the shape of the cusp size distribution is thus the coalescence time $t_{s_{1}, s_{2}}$ of two cells with sizes $s_{1}$ and $s_{2}$, which we determine now.

\section{A. Coalescence time}

We describe the kinetics of disappearance of a node by computing the time it takes for it to travel from its initial location to its nearest neighboring node. This defines the merging, or coalescence time, of two neighboring cells. We call $t_{s_{1}, s_{2}}$ the time it takes to complete the merging according to (10), which reads

$$
\begin{aligned}
t_{s_{1}, s_{2}} & =\int_{0}^{\min \left(s_{1}, s_{2}\right)} \frac{d s^{\prime}}{v\left(s^{\prime}\right)} \\
& =\int_{0}^{s_{2}} \frac{d s^{\prime}}{u\left(\theta_{2}-\theta\right)},
\end{aligned}
$$

if, say, $s_{2}=\min \left(s_{1}, s_{2}\right)$ and where we have made use of (9) in the small $\epsilon=\theta_{2}-\theta$ limit. We denote $\delta$ the height of the cusp above the node level (Fig. 1). It is essentially a constant during the node motion around the symmetrical state, and only vanishes to zero at the very end of the merging process, as seen, for example, in Fig. 2. The node velocity is thus linked to the cusp geometry, and we have

$$
\theta_{2} \approx \tan \theta_{2}=\frac{2 \delta}{s_{2}} \quad \text { and } \quad \theta \approx \tan \theta=\frac{1}{2}=\frac{2 \delta}{\langle s\rangle},
$$

so that the merging time in Eq. (14) writes

$$
\begin{aligned}
t_{s_{1}, s_{2}} & =\frac{\langle s\rangle^{2}}{2 u \delta} \int_{0}^{\zeta} \frac{x d x}{1-x} \\
& \approx \tau \frac{\zeta^{2}}{1-\zeta},
\end{aligned}
$$

with

$$
\tau=\frac{\langle s\rangle}{u} \quad \text { and } \quad \zeta=\frac{s_{2}}{\langle s\rangle} .
$$

Of course, in the singular case of a symmetrical cusp with angles $\theta$ and mean cell size $\langle s\rangle$, the node has no preferred direction to move, and merging does not occur. Consistently, $t_{\langle s\rangle,\langle s\rangle} \rightarrow \infty$ when $\zeta \rightarrow 1$; that was the ideal picture described in Ref. [16]. Given that $\langle s\rangle=2 \pi R_{\mathrm{n}} / N$ and taking 


\section{NODE DYNAMICS AND CUSPS SIZE DISTRIBUTION AT ...}

$N \approx 10$, we find that

$$
\frac{\langle s\rangle}{d}=\frac{\pi \mathrm{We}}{100}, \quad \delta=\frac{\langle s\rangle}{4}, \quad \text { and } \quad \tau \approx \frac{d}{u} \frac{\pi \mathrm{We}}{100},
$$

providing $\langle s\rangle / d \approx 13$ and $\tau \approx 13 \mathrm{~ms}$ for $u=3.07 \mathrm{~m} / \mathrm{s}$ and $d=3 \mathrm{~mm}$ as in Fig. 2, which are consistent orders of magnitude. The cusp height is indeed close to $1 / 4$ the mean cell sizes $\langle s\rangle$, as can be seen from Fig. 1.

\section{B. Mean field coalescence time}

We now compute the mean field coalescence time $t_{\langle s\rangle+\sigma,\langle s\rangle-\sigma}$ of two cells having a size given by the mean size plus or minus a fluctuation of the order of the standard deviation $\sigma$ of the size distribution, as in Eq. (12). Being uneven in size, these cells will merge. Given that the standard deviation of a Gamma distribution of order $v$ is such that $\sigma=\langle s\rangle / \sqrt{v}$, we have, from Eq. (17),

$$
t_{\langle s\rangle+\sigma,\langle s\rangle-\sigma}=\tau \sqrt{\nu}\left(1-\frac{1}{\sqrt{v}}\right)^{2} .
$$

A cell with size $p$ will have time to aggregate with another with size $2\langle s\rangle-p$ to form a cell of size $2\langle s\rangle$ if the typical aggregation time for these two cells is smaller than the aggregation time between two typical cells in the distribution, say, with sizes $\langle s\rangle \pm \sigma$ as in Eq. (20), also producing a cell with size $2\langle s\rangle$ at merging.

The incomplete convolution theory [24] relates the critical cell size $p_{\star}$ below which this criterion is first met, to the order $v$ of the resulting cell size distribution (11) as $p_{\star} /\langle s\rangle=2 / \nu$. Considering two cells with sizes $s_{1}=2\langle s\rangle-2\langle s\rangle / v$ and $s_{2}=2\langle s\rangle / v$ with $s_{2}<s_{1}$, they will coalesce to a size $2\langle s\rangle$ during the typical coalescence time (20) provided

$$
\frac{t_{2\langle s\rangle-2\langle s\rangle / v, 2\langle s\rangle / v}}{t_{\langle s\rangle+\sigma,\langle s\rangle-\sigma}}<1,
$$

so that, making use of Eq. (17), which provides $t_{2\langle s\rangle-2\langle s\rangle / v, 2\langle s\rangle / v}=\tau(2 / \nu)^{2} /(1-2 / \nu)$, and of Eq. (20), we have

$$
\left(\frac{2}{v}\right)^{2}<\sqrt{v}\left(1-\frac{2}{v}\right)\left(1-\frac{1}{\sqrt{v}}\right)^{2}
$$

or

$$
v>4,
$$

a condition which constrains the variance of the cell size distribution. The value $v=4$ is robust: We have shown that the node velocity $v$ depends on the cusp geometry, but if it did not, and was simply a constant with a random orientation, then we would have $t_{s_{1}, s_{2}}=s_{2} / v$ if $s_{2}=\min \left(s_{1}, s_{2}\right)$ so that $t_{\langle s\rangle+\sigma,\langle s\rangle-\sigma}=1-1 / \sqrt{v}$ and $t_{2\langle s\rangle-2\langle s\rangle / v, 2\langle s\rangle / v}=2 / v$, both in units of $\langle s\rangle / v$. The application of the criterion in Eq. (21) would provide the same condition as in Eq. (23).

\section{Cell size distribution}

The ratio in Eq. (21) is a uniformly decreasing function of $v$, and the criterion is first met when $v=4$ (that is, $\sigma /\langle s\rangle=1 / 2)$. Populations of cells more heterogeneous than the critical one $(v<4)$ are therefore not allowed because they live on too long a time scale. More ordered populations around the mean $(v>4)$ are authorized according to $(21)$, but they are, however, metastable since nucleations of new nodes occur randomly along the sheet rim, thus dividing cells into pairs of cells with random sizes. Those complying with the criterion (21) are the only ones liable to survive on a mean coalescence time scale. Node nucleation broadens the cell size distribution, while cell coalescence erases those which are too small, that is, smaller than the critical size $p_{\star} /\langle s\rangle=2 / \nu$, with $v=4$. 
E. VILLERMAUX AND C. ALMARCHA

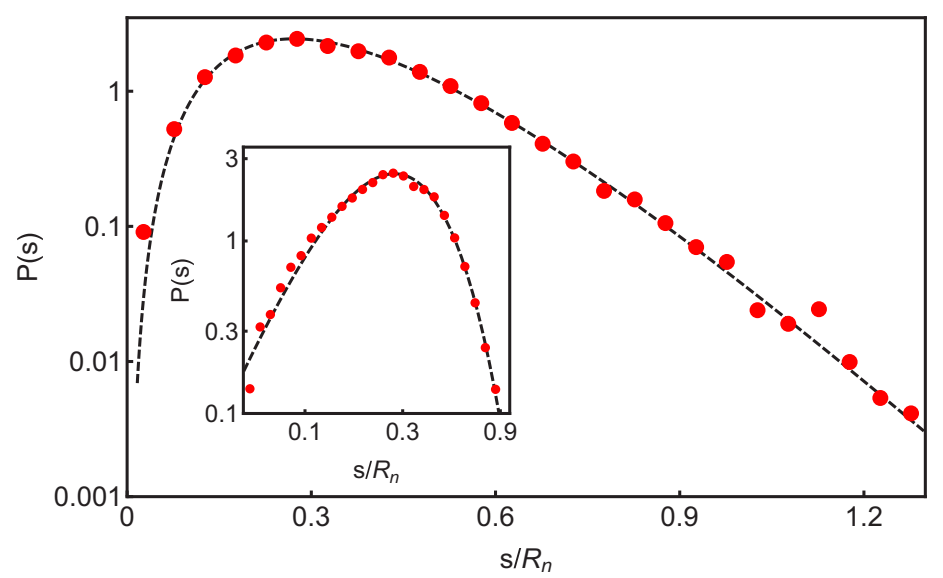

FIG. 5. Statistics of the cell sizes $s$ obtained in the condition of Fig. 1 from the measurement of 47536 independent cusps. The average cell size is such that $\langle s\rangle / R_{\mathrm{n}}=0.36$, corresponding to $N=17$ cusps, in the mean. The dashed line is the Gamma distribution in Eq. (11) with order $v=4$ expected from Eq. (23). Inset: Same in double-logarithmic units (and logarithmic binning), emphasizing the algebraical growth of $P(s)$ at small $s$.

We have processed a large number of instantaneous contours of a Savart sheet at $\mathrm{We}=453$ as in Fig. 1. At every instant of time, with an increment $\Delta t=2 \mathrm{~ms}$, the border of the sheet was extracted according to a procedure similar to the one used for flame fronts [12], nodes were located, and the distance $s$ between consecutive nodes was measured. The corresponding space-time diagram showing how nodes travel, merge, and nucleate is shown in Fig. 4(b). The statistics of the internode distance $s$ is shown in Fig. 5, and compared to the expectation in Eq. (11) with $v=4$.

Although cells nucleate new cusps at random, essentially independently of their size, very big cells, because they are curved, are more likely to divide than standard ones. This may impose a large excursion cutoff in the sizes. This cutoff would, however, affect cells larger than 1.2 times the sheet radius ( $R_{\mathrm{n}}$, to be precise) with an occurrence probability way below 0.01 , as seen in Fig. 5 .

\section{CONCLUSION}

The present problem illustrates a case where global statistics can be understood from detailed microscopic mechanics. The border of liquid sheets is typically indented by a collection of cusps, separated by nodes. Gordillo et al. [16] have shown that the symmetrical stationary cusp is the structure accommodating for both mass and momentum conservation at a steadily receding liquid sheet rim. We have shown here that, if located in between two cusps with different sizes, a node has to move along the border to comply to the same conservation principles. Adapting the reasoning in Ref. [16], the node velocity can be computed as a function of the size contrast between the adjacent cusps. We have further shown how knowledge of this velocity infers the lifetime of adjacent uneven cusps, and the nature of their interaction, which is additive regarding their size.

Our conclusions hold in the self-similar regime where only the Weber number matters, and where we know that the mean cell size scales with We, precisely because the microscopic processes directing the node rearrangements are independent of We, as expressed by Eq. (9). In the $s / R_{\mathrm{n}}$ units, the distribution in Fig. 5 has thus no reason to be different for different We. Liquid viscosity, which does affect the node dynamics, and gravity, if the sheet orientation is not perfectly horizontal, will correct the present picture quantitatively but, most certainly, not qualitatively.

Thus, the indented shape of liquid sheets falls into the category of these fragmented objects whose construction rule is additive, as are scalar mixtures randomly stirred [22], drops formed from 


\section{NODE DYNAMICS AND CUSPS SIZE DISTRIBUTION AT ...}

ligament breakup [23], and broken brittle cohesive solids [24,25], all described by a distribution of the same nature, with parameters reflecting a microscopic determinism.

\section{ACKNOWLEDGMENTS}

We thank José Manuel Gordillo Arias de Saavedra and Henri Lhuissier, with whom the detailed study of cusps started, for insights, Baptiste Néel for assistance in setting up one of the experiments, and the "Agence Nationale de la Recherche" for funding of the ANR "FISICS" No. ANR-15-CE300015-03.

[1] W. Feller, An Introduction to Probability Theory and Its Applications (Wiley, New York, 1970).

[2] F. Savart, Mémoire sur la constitution des Veines liquides lancées par des orifices circulaires en mince paroi, Ann. Chim. 53, 337 (1833).

[3] H. Lhuissier and E. Villermaux, The destabilization of an initially thick liquid sheet edge, Phys. Fluids 23, 091705 (2011).

[4] G. I. Taylor, The dynamics of thin sheets of fluid. III. Disintegration of fluid sheets, Proc. R. Soc. London, Ser. A 253, 313 (1959).

[5] J. C. P. Huang, The break-up of axisymmetric liquid sheets, J. Fluid Mech. 43, 305 (1970).

[6] C. Clanet and E. Villermaux, Life of a smooth liquid sheet, J. Fluid Mech. 462, 307 (2002).

[7] C. Ellegaard, A. E. Hansen, A. Haaning, K. Hansen, A. Marcussen, T. Bohr, J. L. Hansen, and S. Watanabe, Creating corners in kitchen sinks, Nature (London) 392, 767 (1998).

[8] R. Buckingham and J. W. M. Bush, Fluid polygons, Phys. Fluids 13, S10 (2001).

[9] H. Lhuissier and E. Villermaux, Crumpled water bells, J. Fluid Mech. 693, 508 (2012).

[10] J. O. Marston, T. T. Truscott, N. B. Spiers, M. M. Mansoor, and S. T. Thoroddsen, Crown sealing and buckling instability during water entry of spheres, J. Fluid Mech. 794, 506 (2016).

[11] Y. D'Angelo, G. Joulin, and G. Boury, On model evolution equations for the whole surface of threedimensional expanding wrinkled premixed flames, Combust. Theory Modell. 4, 317 (2000).

[12] C. Almarcha, J. Quinard, B. Denet, E. Al-Sarraf, J. M. Laugier, and E. Villermaux, Experimental two dimensional cellular flames, Phys. Fluids 27, 091110 (2015).

[13] E. Dressaire, L. Courbin, A. Delancy, M. Roper, and H. A. Stone, Study of polygonal water bells: Inertia-dominated thin-film flows over microtextured surfaces, J. Fluid Mech. 721, 46 (2013).

[14] E. Villermaux, V. Pistre, and H. Lhuissier, The viscous Savart sheet, J. Fluid Mech. 730, 607 (2013).

[15] H. Lhuissier, B. Néel, and L. Limat, Viscoelasticity Breaks the Symmetry of Impacting Jets, Phys. Rev. Lett. 113, 194502 (2014).

[16] J. M. Gordillo, H. Lhuissier, and E. Villermaux, On the cusps bordering liquid sheets, J. Fluid Mech. 754, R1 (2014).

[17] A. Dupré, Théorie mécanique de la chaleur, Ann. Chim. Phys. 11, 194 (1868).

[18] L. Rayleigh, Some applications of photography, Nature (London) 44, 249 (1891).

[19] P. G. de Gennes, Mechanics of soft interfaces, Faraday Discuss. 104, 1 (1996).

[20] H. Lhuissier and E. Villermaux, Destabilization of flapping sheets: The surprising analog of soap films, C. R. Mec. 337, 469 (2009).

[21] F. E. C. Culick, Comments on a ruptured soap film, J. Appl. Phys. 31, 1128 (1960).

[22] J. Duplat and E. Villermaux, Mixing by random stirring in confined mixtures, J. Fluid Mech. 617, 51 (2008).

[23] E. Villermaux, Fragmentation, Annu. Rev. Fluid Mech. 39, 419 (2007).

[24] A. Vledouts, N. Vandenberghe, and E. Villermaux, Fragmentation as an aggregation process, Proc. R. Soc. London, Ser. A 471, 20150678 (2015).

[25] A. Vledouts, N. Vandenberghe, and E. Villermaux, Fragmentation as an aggregation process: The role of defects, Proc. R. Soc. London, Ser. A 472, 20150679 (2016). 